Pacific Journal of Mathematics

PRODUCTS OF POSITIVE DEFINITE MATRICES. II 


\section{PRODUCTS OF POSITIVE DEFINITE MATRICES. II}

\section{S. Ballantine}

This paper is concerned with the problem of determining, for given positive integers $n$ and $j$, which $n \times n$ matrices (of positive determinant) can be written as a product of $j$ positive definite matrices. In $\S 2$ the $2 \times 2$ complex case is completely solved. In particular, it turns out that every $2 \times 2$ complex matrix of positive determinant can be factored into a product of five positive definite Hermitian matrices and, unless it is a negative scalar matrix, can even be written as a product of four positive definite matrices. Sections 3 and 4 deal with the general $n \times n$ case. In $\S 3$ it is shown that a scalar matrix $\lambda I$ can be written as a product of four positive definite Hermitian matrices only if the scalar $\lambda$ is real and positive, and that $\lambda H$ ( $\lambda$ complex, $H$ Hermitian) can be written as a product of three positive definite matrices only if $\lambda H$ is itself positive definite. In $\S 4$ it is shown that every $n \times n$ real matrix of positive determinant can be written as a product of six positive definite real symmetric matrices and that every $n \times n$ complex matrix of positive determinant can be written as a product of eleven positive definite Hermitian matrices.

The $2 \times 2$ real case was earlier solved in $[1$, Th. 1 and the remarks immediately following]. The results in $\S 4$ use only the $2 \times 2$ results and certain well known results. In later papers of this series the results of $\S 4$ will be improved upon, using more refined methods.

In the rest of this section we state without proof several well known results that we shall use in later sections. First we introduce some notation. For a fixed positive integer $n$ we denote by $\mathscr{F}$ the set of all $n \times n$ Hermitian matrices and by $\mathscr{P}$ the set of all positive definite matrices in $\mathscr{H}$. Then for each positive integer $j$ we denote by $\mathscr{S}^{j}$ the set consisting of every $n \times n$ complex matrix which can be written as a product of $j$ matrices from $\mathscr{P}$. (Thus $\mathscr{P}^{1}=\mathscr{P}^{\text {.) }}$ Analogously, we denote by $\mathscr{K}$ the set of all $n \times n$ real symmetric matrices (thus $\mathscr{K}$ is just the set of all real matrices of $\mathscr{H}$ ), by $\mathscr{R}$ the set of all positive definite matrices of $\mathscr{K}$ (thus $\mathscr{R}$ is just the set of all real matrices of $\mathscr{P}$ ), and by $\mathscr{R}^{j}$ the set consisting of every $n \times n$ real matrix which can be written as a product of $j$ matrices from $\mathscr{R}$ (so $\mathscr{R}^{1}=\mathscr{R}$ ). For a matrix $S$ all of whose eigenvalues are real the inertia of $S$ is the ordered triple (of nonnegative integers) consisting of the number of positive eigenvalues 
of $S$, the number of negative eigenvalues of $S$, and the nullity of $S$ [3, p. 1119].

The following theorem is essentially Sylvester's Inertia Theorem combined with standard diagonalization theorems, but in a form convenient for our present purposes [e.g., 3, Corollary 8, p. 1127].

Theorem 1. Let $H \in \mathscr{P}$ and $P \in \mathscr{P}$. Then $H P$ is similar over the complex field to some $K \in \mathscr{H}$, and the inertia of $K$ is necessarily the same as that of $H$. Conversely, if $S$ is similar to some $K \in \mathscr{H}$ then there are matrices $H \in \mathscr{H}$ and $P \in \mathscr{P}$ such that $S=H P$, and $H$ and $K$ (and $S$ ) necessarily have the same inertia.

Specializing to the case when $H$ is itself in $\mathscr{P}$, we obtain the following:

CoROLlary 1.1. $S \in \mathscr{P}^{2}$ if and only if $S$ is similar over the complex field to an element of $\mathscr{P}$, i.e., if and only if $S$ is diagonable and has only positive eigenvalues.

The real analogs of the above theorem and corollary also hold. Of these we shall need only the latter, which we now state.

CoROLlaRY 1.1'. $S \in \mathscr{R}^{2}$ if and only if $S$ is similar over the real field to an element of $\mathscr{R}$, i.e., if and only if $S$ is real and diagonable and has only positive eigenvalues.

We conclude this section with three obvious facts about $\mathscr{P}^{j}$ and $\mathscr{R}^{j}$.

Fact 1. $\mathscr{P}^{j}$ is invariant under unitary similarity, that is, $S \in \mathscr{P}^{j}$ implies every matrix unitarily similar to $S$ is also $\in \mathscr{P}^{j}$. (Analogously, $\mathscr{R}^{j}$ is invariant under orthogonal similarity.)

Fact 2. $\mathscr{P}^{j}$ is invariant under multiplication by positive scalars (as is $\mathscr{R}^{j}$ ), that is, $S \in \mathscr{P}^{j}$ implies $\lambda S \in \mathscr{P}^{j}$ for every $\lambda>0$.

Fact 3. $\mathscr{P}^{j}$ is invariant under inversion (as is $\mathscr{R}^{j}$ ), that is, $S \in \mathscr{P}^{j}$ implies $S^{-1} \in \mathscr{P}^{j}$.

2. The $2 \times 2$ complex case. In this section we start by assuming $S$ is a $2 \times 2$ complex matrix with $\operatorname{det} S>0$. We shall derive necessary and sufficient conditions (nasc) on $S$ that $S$ be $\in \mathscr{P}^{3}$ (these nasc are given in Theorem 2 below). Now, $S \in \mathscr{P}^{3}$ if and only if there is a $P \in \mathscr{P}$ such that $P S \in \mathscr{P}^{2}$. By Fact $2(\S 1)$ we may assume 
$S$ is unimodular and need only consider unimodular solutions $P$. It is well known that every square complex matrix is unitarily similar to a lower triangular matrix [4, p. 165, Corollary 6.2, or 5, p. 229, Th. 8.14 (applied to the transposed conjugate matrix)], so we may further (by Fact 1 ) assume that $S$ is lower triangular, i.e., that

$$
S=\left[\begin{array}{cc}
p e^{i \theta} & 0 \\
q & p^{-1} e^{-i \theta}
\end{array}\right]
$$

where $p>0, \theta$ is real, and (by performing a further diagonal unitary similarity if necessary) $q \geqq 0$. We want usable nasc on $P S$ that it be $\in \mathscr{P}^{2}$, so we use the following obvious consequence of Corollary 1.1: Let $a d-b c=1$. Then the (unimodular) matrix

$$
T=\left[\begin{array}{ll}
a & b \\
c & d
\end{array}\right]
$$

is $\epsilon \mathscr{P}^{2}$ if and only if $T=I$ or $a+d$ is real and $>2$. Now we let

$$
P=\left[\begin{array}{ll}
r & \bar{z} \\
z & t
\end{array}\right]
$$

so that $r>0$, and $r t-\bar{z} z=1$. We shall next put $z=x+i y$, with $x$ and $y$ real. Thus $S \in \mathscr{P}^{3}$ if and only if the following system has an admissible (real) solution $(r, t, x, y)$ (i.e., a solution in which $r>0$ and $t>0)$ :

$$
\begin{aligned}
\operatorname{Im}\{\operatorname{trace}(P S)\} & \equiv\left(r p-t p^{-1}\right) \sin \theta-y q=0 \\
\operatorname{Re}\{\operatorname{trace}(P S)\} & \equiv\left(r p+t p^{-1}\right) \cos \theta+x q>2 \\
\quad 4(r t-\bar{z} z) & \equiv\left(r p+t p^{-1}\right)^{2}-\left(r p-t p^{-1}\right)^{2}-4 x^{2}-4 y^{2}=4 .
\end{aligned}
$$

(We have here seemingly ignored the possibility that $P S=I$, but if $P S$ were $=I$ we would have $S$ itself $\in \mathscr{P}$ (and hence $q$ would $=0$ and $e^{i \theta}$ would $=1$ ) and the above system would then certainly have an admissible solution.)

The grouping of terms in the above system suggests the substitution

$$
u=r p+t p^{-1}, v=r p-t p^{-1}
$$

which (for fixed $p>0$ ) is a nonsingular linear substitution taking the admissible region

$$
\{(r, t): r>0, t>0\}
$$

onto the new admissible region 


$$
\{(u, v): u>|v|\}
$$

in the new unknowns $u, v$. Thus $S \in \mathscr{P}^{3}$ if and only if the following system has an admissible solution (for $u, v, x, y$, in which $u>|v|$ ):

$$
\begin{gathered}
v \sin \theta-y q=0 \\
u \cos \theta+x q>2 \\
u^{2}=4\left(1+x^{2}+y^{2}\right)+v^{2} .
\end{gathered}
$$

Clearly, if this system has any admissible solution at all, then it has an admissible solution with $x \geqq 0$ (simply replace $x$ by $|x|$ in the solution), and hence has an admissible solution in which $y=v=0$ and in which $u$ has its former value and $x$ has a value $\geqq$ its former value (now assumed $\geqq 0$ ) in the original solution. Thus $S \in \mathscr{P}^{3}$ if and only if the following system has an admissible solution (for $u$ and $x$, with $u>0$ and $x \geqq 0$ ):

$$
\begin{aligned}
& u \cos \theta+x q>2 \\
& u=2\left(1+x^{2}\right)^{1 / 2} .
\end{aligned}
$$

This last system evidently has an admissible solution if and only if the inequality

$$
2\left(1+x^{2}\right)^{1 / 2} \cos \theta+x q>2
$$

has a solution $x \geqq 0$. In order to see more easily what happens as $x \rightarrow+\infty$, we rewrite this inequality as follows:

$$
(2 \cos \theta+q)\left(1+x^{2}\right)^{1 / 2}+q\left[x-\left(1+x^{2}\right)^{1 / 2}\right]>2 .
$$

Now, if $2 \cos \theta+q \leqq 0$, then the left side of (1) is $\leqq 0$ for all real $x$ (since $q \geqq 0$ ) and hence (1) has no solution. On the other hand, if $2 \cos \theta+q>0$, then all sufficiently large $x$ are (admissible) solutions of (1) since as $x \rightarrow+\infty$ the first term on the left $\rightarrow+\infty$ while the second term is bounded below.

Thus we arrive at the preliminary result that $S \in \mathscr{P}^{3}$ if and only if $2 \cos \theta>-q$ (where $p>0, q \geqq 0$ always). We first square out and get

$$
q^{2}>4 \cos ^{2} \theta \quad \text { when } \cos \theta \leqq 0
$$

for our nasc on $S$. Now, $q^{2}$ and $\cos \theta$ are unitary invariants of $S$ but are not rational unitary invariants. Thus we pick a system of rational unitary invariants and express the above nasc in terms of this system. In terms of a "general" $2 \times 2$ matrix $T$ (not necessarily unimodular), one such system would be 
(where $T^{*}$ means the transposed conjugate of $T$ ). In the present case we have (replacing $T$ by $S$ )

$$
\begin{gathered}
\operatorname{det} S=1 \\
\operatorname{trace} S=p e^{i \theta}+p^{-1} e^{-i \theta}=\left(p+p^{-1}\right) \cos \theta+i\left(p-p^{-1}\right) \sin \theta \\
\operatorname{trace}\left(S^{*} S\right)=p^{2}+p^{-2}+q^{2} .
\end{gathered}
$$

A straightforward calculation (which could be quite lengthy if undertaken without foreknowledge of the result) reveals that

$$
q^{2}-4 \cos ^{2} \theta=\operatorname{trace}\left(S^{*} S\right)-|\operatorname{trace} S|^{2}-2 \text {. }
$$

Also, by inspection we obviously have

$$
\cos \theta \leqq 0 \text { if and only if } \operatorname{Re}\{\text { trace } S\} \leqq 0 .
$$

Thus our nase on $S$ (that $S$ be $\in \mathscr{T}^{3}$ ) are

$$
\operatorname{tr}\left(S^{*} S\right)-|\operatorname{tr} S|^{2}-2>0 \text { whenever } \operatorname{Re}\{\operatorname{tr} S\} \leqq 0
$$

(where tr means trace). This condition describes a unitarily invariant set of unimodular matrices, which set (by our derivation above) contains all lower triangular unimodular matrices of $\mathscr{S}^{3}$ and contains no lower triangular unimodular marices that are not $\in \mathscr{P}^{3}$. Thus (by Fact 1) it gives nasc on an arbitrary unimodular matrix $S$ that $S$ be $\in \mathscr{P}^{3}$. If now $S$ is an arbitrary $2 \times 2$ matrix of determinant $\Delta>0$, then we apply the above condition to the unimodular matrix $\Delta^{-(1 / 2)} S$, and get

$$
\operatorname{tr}\left(S^{*} S\right)-|\operatorname{tr} S|^{2}-2 \Delta>0 \text { whenever } \operatorname{Re}\{\operatorname{tr} S\} \leqq 0 .
$$

This is therefore (by Fact 2 ) a nasc that an arbitrary $2 \times 2$ complex matrix $S$ be $\in \mathscr{S}^{3}$, and this (plus a routine simplification) proves the second conclusion in the following theorem.

TheOREm 2. Let $a, b, c, d$ be complex numbers such that $a d-b c>0$. Then the matrix

$$
\left[\begin{array}{ll}
a & b \\
c & d
\end{array}\right]
$$

is $\in \mathscr{P}^{2}$ if and only if

$$
a+d \text { is real and }>2(a d-b c)^{1 / 2} \text {, or else } b=c=0 \text { and } a=d>0 \text {; }
$$

is $\in \mathscr{S}^{3}$ if and only if

$$
|\bar{c}-b|^{2}+(a-\bar{a})(d-\bar{d})>4(a d-b c) \quad \text { whenever } \operatorname{Re}\{a+d\} \leqq 0 ;
$$


is $\in \mathscr{P}^{4}$ if and only if

it is not a negative scalar matrix;

is $\in \mathscr{P}^{5}$ (always).

Proof. The nasc for $\mathscr{P}^{2}$ is just the "homogenized" version of the condition given near the beginning of this section for unimodular matrices. (It is well known, but is given here for purposes of comparison.) We could prove the nasc for $\mathscr{P}^{4}$ the same way we proved the nasc for $\mathscr{P}^{3}$ (i.e., by derivation), but this method would be unnecessarily laborious. (The result itself is easily conjectured by analogy with the real case [1, loc. cit.].) The "only if" part of the $\mathscr{P}^{4}$ nasc is a special case of Theorem 3, which is stated and proved in the next section. For the "if" part, assume $S$ is not a negative scalar matrix. We may also assume $S$ is not a positive scalar matrix (if it were, it would obviously be $\in \mathscr{P}^{4}$ ). Then $S$ is unitarily similar to a nondiagonal matrix. (E.g., if $S=\operatorname{diag}(l, m)$ with $l \neq m$, and $U$ is the matrix of a $45^{\circ}$-rotation, then $U^{-1} S U$ has $\pm(1 / 2)(l-m) \neq 0$ in the off-diagonal positions.) Thus (by Fact 1 ) we may assume $S$ is nondiagonal:

$$
S=\left[\begin{array}{ll}
a & b \\
c & d
\end{array}\right], \quad(c, b) \neq(0,0)
$$

(and always $a d-b c>0$ ). We have only to show that there is a $P \in \mathscr{P}$ such that $P S \in \mathscr{P}^{3}$. We shall show this, in fact with a diagonal $P$. Let $P=\operatorname{diag}(r, t)$ with $r>0$ and $t>0$. Then, by the nasc for $\mathscr{P}^{3}, P S \in \mathscr{P}^{3}$ if

$$
|t \bar{c}-r b|^{2}+r t(a-\bar{a})(d-\bar{d})-4 r t(a d-b c)>0 .
$$

If $c \neq 0$, we can take $t=1$, and then obviously we can take $r$ sufficiently small (but $>0$ ) to satisfy the above inequality; if $c=0$, then $b \neq 0$ and we take $r=1$ and $t$ sufficiently small.

To prove the last conclusion (of Theorem 2) it suffices to show that every negative scalar matrix is $\in \mathscr{P}^{5}$. This already follows from the real case [1, loc. cit.], but we can give also a brief independent proof here. Suppose $S$ is a negative scalar matrix. Then for any nonscalar $P \in \mathscr{P} P S$ is nonscalar (and has positive determinant), so (by the $\mathscr{P}^{4}$ nasc) $P S \in \mathscr{P}^{4}$, and hence $S \in \mathscr{P}^{5}$.

REMARK 1. The above proof can be adapted to yield a second proof for the real case (proved in [1, loc. cit.] by a completely different method). No essential modification would be needed in the proofs of the $\mathscr{P}^{4}$ and $\mathscr{P}^{5}$ parts to get the respective proofs of the $\mathscr{R}^{4}$ and 
$\mathscr{R}^{5}$ results. For the $\mathscr{R}^{3}$ proof we could use the fact that each $2 \times 2$ real matrix is orthogonally similar to one of the form

$$
\left[\begin{array}{rr}
a & -c \\
c & d
\end{array}\right]
$$

[6, p. 185, Example 39] and proceed from there as in the above proof of the $\mathscr{T}^{3}$ nasc. This same method could also be used (in the $2 \times 2$ case) to derive the respective nasc's for $\mathscr{S}^{2}$ and $\mathscr{R}^{2}$ directly (without using Corollaries 1.1 or $\left.1.1^{\prime}\right)$.

REMARK 2. One might imagine using this same method (the one used above to derive the $\mathscr{P}^{3}$ nasc when $n=2$ ) to derive the corresponding results (or prove the results after correctly conjecturing them) for $\mathscr{P}^{3}$ or $\mathscr{R}^{3}$ when $n \geqq 3$. However, prospects in this direction are decidedly unpromising.

REMARK 3. Since $\mathscr{R} \subset \mathscr{P}$ it is obvious that $\mathscr{R}^{j} \subset \mathscr{P}^{j}$ for each $j$, but it is not a priori evident that for $j \geqq 2 \mathscr{R}^{j}$ contains all the real matrices of $\mathscr{P}^{j}$. However, comparison of the real and complex $2 \times 2$ results reveals that when $n=2 \mathscr{R}^{j}$ does indeed contain all the real matrices of $\mathscr{P}^{j}$ (for all $j$ ). Also, it is known that, for arbitrary $n, \mathscr{R}^{2}$ contains all the real matrices of $\mathscr{P}^{2}$, since this follows from Corollaries 1.1 and $1.1^{\prime}$ and standard diagonalization theorems, e.g. [4, p. 101, Th. 7.4, or 5, p. 180, Th. 7.5].

3. Some nonexistent factorizations $(n \times n$ case). Turning now to the general $n \times n$ case, we shall show that only the obvious scalar matrices are $\in \mathscr{P}^{4}$ (viz., the positive ones), and that only the obvious Hermitian matrices (or, more generally, only the obvious scalar multiples of Hermitian matrices) are $\in \mathscr{P}^{3}$ (viz., those that are $\in \mathscr{P}$ ). The analogous real results follow immediately, so we shall not state them separately.

THEOREM 3. Let $\lambda$ be a complex scalar and $I$ be the $n \times n$ identity matrix. Then $\lambda I \in \mathscr{P}^{4}$ only if $\lambda>0$.

Proof. Suppose $\lambda I \in \mathscr{P}^{4}$. Then there are $S_{1}$ and $S_{2}$, both $\in \mathscr{P}^{2}$, such that $\lambda I=S_{1} S_{2}$. Thus

$$
\lambda S_{1}^{-1}=S_{2}
$$

and (by Fact 3) $S_{1}^{-1} \in \mathscr{P}^{2}$. Now, by Corollary 1.1 all the eigenvalues of $S_{1}^{-1}$ and of $S_{2}$ are positive, so all those of $\lambda S_{1}^{-1}$ have signum $=\operatorname{sgn} \lambda$, hence by (2) $\operatorname{sgn} \lambda=1$, i.e., $\lambda>0$. 
THEOREM 4. Let $\lambda$ be a complex scalar and $H$ be a complex Hermitian $n \times n$ matrix. Then $\lambda H \in \mathscr{P}^{3}$ only if $\lambda H \in \mathscr{P}$.

Proof. Suppose $\lambda H \in \mathscr{S}^{3}$. Then there are $S \in \mathscr{P}^{2}$ and $P \in \mathscr{P}$ such that $\lambda H=S P$. Thus

$$
\lambda H P^{-1}=S
$$

and (by Fact 3) $P^{-1} \in \mathscr{S}$. Now, by Corollary 1.1 all the eigenvalues of $S$ are positive and by Theorem $1 H^{-1}$ is similar to a Hermitian matrix, hence has only real eigenvalues, so all eigenvalues of $\lambda H P^{-1}$ have signa $= \pm \operatorname{sgn} \lambda$. Therefore by $(3) \lambda$ is real and all eigenvalues of $\lambda H P^{-1}$ are positive, so by Theorem 1 (since $\lambda H$ is Hermitian) all eigenvalues of $\lambda H$ are positive. Thus $\lambda H$ is positive definite Hermitian.

4. Some factorizations which do exist $(n \times n$ case). In the last section we showed that two special classes of matrices had no members in, respectively, $\mathscr{P}^{3}$ and $\mathscr{P}^{4}$; the classes were chosen the way they were, partly in order to minimize the proofs. In this section we shall show that there is a $j$, independent of $n$, such that every complex $n \times n$ matrix of positive determinant is $\in \mathscr{P}^{j}$, and here we shall choose $j$ in a way which minimizes the proof. We shall derive the same result for the real case, except with a considerably smaller value of $j$.

We start with the real case. By [1, loc. cit.] we know that every $2 \times 2$ rotation matrix is $\in \mathscr{R}^{5}$ ("rotation matrix" means "unimodular real orthogonal matrix"). Now, it is well known [6, pp. 109-110, Example 14] that each $n \times n$ rotation matrix is orthogonally similar to a block-diagonal matrix each of whose diagonal blocks is a $2 \times 2$ rotation matrix, except when $n$ is odd there is also one $1 \times 1$ diagonal block (consisting of a 1 ).

THEOREM 5. Let $R$ be an $n \times n$ rotation matrix. Then

(i) $R \in \mathscr{R}^{5}$;

(ii) if all of the real eigenvalues of $R$ are positive, $R \in \mathscr{R}^{4}$;

(iii) if all eigenvalues of $R$ have positive real parts, $R \in \mathscr{R}^{3}$; and

(iv) if $R \neq I, R \notin \mathscr{R}^{2}$.

Proof. By Fact 1 we need only consider the case where $R$ is in the block-diagonal form described above. Then the first three parts follow from the corresponding $2 \times 2$ results ([1], loc. cit., or [2], Th. 2.4 and the second paragraph following it, pp. 270-271, where the $2 \times 2$ rotation matrices are discussed separately). Part (iv) follows 
from Corollary 1.1'.

COROLlary 5.1. Each $n \times n$ real matrix of positive determinant is $\in \mathscr{R}^{6}$.

Proof. Let $S$ be a real $n \times n$ matrix and $\operatorname{det} S>0$. Then [6, p. 185, Examples 41-42] there are matrices $P$ and $R$ such that $P \in \mathscr{R}, R$ is real orthogonal, and $S=P R$. Thus $\operatorname{det} R>0$ and hence $R$ is a rotation matrix and so (by (i) of Theorem 5) is $\in \mathscr{R}^{5}$. Therefore $S \in \mathscr{R}^{6}$.

REMARK. For $n \geqq 3$ the converses of (ii) and (iii) of Theorem 5 are false, as will be shown by the following examples. For our first example,

$$
\left[\begin{array}{rrr}
0 & -1 & 0 \\
1 & 0 & 0 \\
0 & 0 & 1
\end{array}\right]=\frac{1}{4}\left[\begin{array}{ccc}
2 & 0 & 0 \\
0 & 7 & -3 x \\
0 & -3 x & 7
\end{array}\right]\left[\begin{array}{rrr}
10 & -9 & -6 x \\
-9 & 10 & 6 x \\
-6 x & 6 x & 19
\end{array}\right]\left[\begin{array}{rrr}
27 & -20 & 15 x \\
-20 & 18 & -12 x \\
15 x & -12 x & 43
\end{array}\right],
$$

where $x=\sqrt{5}$. The three factors on the right are $\in \mathscr{R}$, while their product (which is $\in \mathscr{R}^{3}$ ) is a matrix of a (90-degree) rotation and (thus) two of its eigenvalues have real part $=0$. Therefore the converse of (iii) is false.

For our second example,

$$
\begin{gathered}
{\left[\begin{array}{rrr}
-1 & 0 & 0 \\
0 & -1 & 0 \\
0 & 0 & 1
\end{array}\right]=\frac{1}{4}\left[\begin{array}{llr}
1 & 0 & 0 \\
0 & 4 & -x y \\
0 & -x y & 4
\end{array}\right]\left[\begin{array}{rrr}
5 & 0 & -x z \\
0 & 2 & 0 \\
-x z & 0 & 5
\end{array}\right]} \\
\times\left[\begin{array}{cccc}
29 & 6 y z & 10 x z \\
6 y z & 46 & 15 x y \\
10 x z & 15 x y & 74
\end{array}\right]\left[\begin{array}{ccc}
65 & 15 y z & -24 x z \\
15 y z & 127 & -40 x y \\
-24 x z & -40 x y & 190
\end{array}\right],
\end{gathered}
$$

where $x=\sqrt{3}, y=\sqrt{5}, z=\sqrt{7}$. The four factors on the right are $\in \mathscr{R}$, while their product (which is $\left.\in \mathscr{R}^{4}\right)$ is a (180-degree) rotation matrix with two negative real eigenvalues. Therefore the converse of (ii) is false.

We now turn to the complex case. It is well known [4, p. 172, Th. 9.2] that each $n \times n$ unitary matrix is unitarily similar to a diagonal (unitary) matrix (all of whose diagonal entries necessarily have absolute value unity). Thus it suffices (by Fact 1) to consider the diagonal case. By Theorem 2 (last part) we know that every $2 \times 2$ unimodular unitary matrix is $\in \mathscr{P}^{5}$, but, when $n \geqq 3$, an $n \times n$ diagonal unimodular unitary matrix $U$ ordinarily will not have its diagonal entries occurring in conjugate pairs (even those 
diagonal entries that are not positive), so we cannot proceed so directly as we did in the real case. What we can do is write $U=U_{1} U_{2}$, where the diagonal entries of $U_{1}$ occur in conjugate pairs (except for an extra 1 when $n$ is odd) and likewise for $U_{2}$. One such way to factor $U$ is as follows. Suppose

$$
U=\operatorname{diag}\left(u_{1}, u_{2}, \cdots, u_{n}\right)
$$

where $u_{j} \bar{u}_{j}=1$ for all $j$ and $\operatorname{det} U=u_{1} u_{2} \cdots u_{n}=1$. Then we let

$$
\begin{aligned}
& U_{1}=\operatorname{diag}\left(u_{1}, \bar{u}_{1}, u_{1} u_{2} u_{3}, \bar{u}_{1} \bar{u}_{2} \bar{u}_{3}, \cdots\right), \\
& U_{2}=\operatorname{diag}\left(1, u_{1} u_{2}, \bar{u}_{1} \bar{u}_{2}, u_{1} u_{2} u_{3} u_{4}, \cdots\right),
\end{aligned}
$$

where the respective last (i.e., $n$ th) diagonal entries are $u_{n}$ and 1 if $n$ is even, and 1 and $u_{n}$ if $n$ is odd. Now we can apply the last part of Theorem 2 to each of the $2 \times 2$ blocks

$$
\operatorname{diag}\left(u_{1}, \bar{u}_{1}\right), \operatorname{diag}\left(u_{1} u_{2} u_{3}, \bar{u}_{1} \bar{u}_{2} \bar{u}_{3}\right), \cdots
$$

of $U_{1}$ and to each of the $2 \times 2$ blocks

$$
\operatorname{diag}\left(u_{1} u_{2}, \bar{u}_{1} \bar{u}_{2}\right), \cdots
$$

of $U_{2}$, and can conclude that each such block is $\in \mathscr{P}^{5}$. Thus $U$ itself is $\in \mathscr{P}^{10}$, and we have proved:

Theorem 6. Each $n \times n$ unimodular unitary matrix is $\in \mathscr{P}^{10}$.

COROLLARY 6.1. Each $n \times n$ complex matrix of positive determinant is $\in \mathscr{P}^{11}$.

Proof. The proof is entirely analogous to that of Corollary 5.1.

Remark. The exponents occurring in Corollary 5.1, Theorem 6, and Corollary 6.1 ("six", "ten", and "eleven", respectively) can be reduced (to "five", "five", and "five", respectively, and, by Theorem 3 , no further (if $n \geqq 2$ )) by using more refined methods. We shall employ such methods in the next papers in this series.

\section{REFERENCES}

1. C. S. Ballantine, Products of positive definite matrices. I, Pacific J. Math.

2. —_ A semigroup generated by definite matrices, J. d'Analyse Math. 12 (1964), 257-275.

3. David H. Carlson, On real eigenvalues of complex matrices, Pacific J. Math. 15 (1965), 1119-1129.

4. Evar D. Nering, Linear Algebra and Matrix Theory, John Wiley, New York, 1963. 
5. Robert R. Stoll, Linear Algebra and Matrix Theory, McGraw-Hill, New York, 1952.

6. H. W. Turnbull and A. C. Aitken, An Introduction to the Theory of Canonical Matrices, Blackie, Glasgow, 1932.

Received December 6, 1966.

OREgon State UNIVERSITY 



\section{PACIFIC JOURNAL OF MATHEMATICS}

\section{EDITORS}

\section{H. ROYDEN}

Stanford University

Stanford, California

\section{J. P. Jans}

University of Washington

Seattle, Washington 98105

\section{J. DUGUNDJI}

Department of Mathematics

Rice University

Houston, Texas 77001

RICHARD ARENS

University of California

Los Angeles, California 90024

\section{ASSOCIATE EDITORS}
E. F. BeCKenbaCH
B. H. NeumanN
F. WOLF
K. YosIDA

\section{SUPPORTING INSTITUTIONS}

UNIVERSITY OF BRITISH COLUMBIA CALIFORNIA INSTITUTE OF TECHNOLOGY UNIVERSITY OF CALIFORNIA MONTANA STATE UNIVERSITY UNIVERSITY OF NEVADA NEW MEXICO STATE UNIVERSITY OREGON STATE UNIVERSITY UNIVERSITY OF OREGON OSAKA UNIVERSITY UNIVERSITY OF SOUTHERN CALIFORNIA
STANFORD UNIVERSITY UNIVERSITY OF TOKYO UNIVERSITY OF UTAH WASHINGTON STATE UNIVERSITY UNIVERSITY OF WASHINGTON

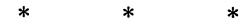

AMERICAN MATHEMATICAL SOCIETY CHEVRON RESEARCH CORPORATION TRW SYSTEMS

NAVAL ORDNANCE TEST STATION 


\section{Pacific Journal of Mathematics}

\section{Vol. 24, No. $1 \quad$ May, 1968}

Harry P. Allen, Lie algebras of type $D_{4}$ over algebraic number fields ...... 1

Charles Ballantine, Products of positive definite matrices. II............ 7

David W. Boyd, The spectral radius of averaging operators ............ 19

William Howard Caldwell, Hypercyclic rings ................... 29

Francis William Carroll, Some properties of sequences, with an application

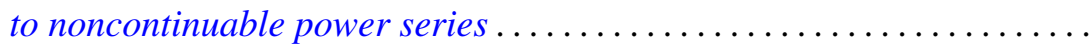

David Fleming Dawson, Matrix summability over certain classes of

sequences ordered with respect to rate of convergence ........... 51

D. W. Dubois, Second note on David Harrison's theory of preprimes. . . . . 57

Edgar Earle Enochs, A note on quasi-Frobenius rings.............. 69

Ronald J. Ensey, Isomorphism invariants for Abelian groups modulo bounded groups ................................ 71

Ronald Owen Fulp, Generalized semigroup kernels ................ 93

Bernard Robert Kripke and Richard Bruce Holmes, Interposition and approximation ................................. 103

Jack W. Macki and James Sai-Wing Wong, Oscillation of solutions to second-order nonlinear differential equations ..................

Lothrop Mittenthal, Operator valued analytic functions and generalizations

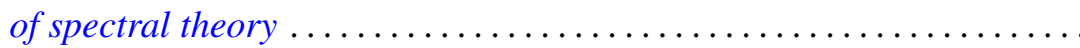

T. S. Motzkin and J. L. Walsh, A persistent local maximum of the pth power deviation on an interval, $p<1 \ldots \ldots \ldots \ldots \ldots \ldots \ldots \ldots \ldots \ldots . \ldots \ldots$

Jerome L. Paul, Sequences of homeomorphisms which converge to homeomorphisms ...........................

Maxwell Alexander Rosenlicht, Liouville's theorem on functions with elementary integrals.

Joseph Goeffrey Rosenstein, Initial segments of degrees .

$\mathrm{H}$. Subramanian, Ideal neighbourhoods in a ring ............

Dalton Tarwater, Galois cohomology of abelian groups . .

James Patrick Williams, Schwarz norms for operators ... .

Raymond Y. T. Wong, A wild Cantor set in the Hilbert cube. 